\title{
Low Power Transmission for Autonomous Wireless Indoor Sensors
}

\author{
A. A. Valenzuela ${ }^{1}$, H. N. Abdullah ${ }^{2}$ \\ ${ }^{1}$ Bonn-Rhein-Sieg University of Applied Sciences, Sankt Augustin, Germany, \\ alejandro.valenzuela@h-brs.de \\ ${ }^{2}$ Al-Mustansiryah University, Baghdad, Iraq
}

\begin{abstract}
:
Cost efficient energy monitoring in existing large buildings demands for autonomous indoor sensors with low power consumption, high performance in multipath fading channels and economic implementation. Good performance in multipath fading channels can be achieved with noncoherent chaotic modulation schemes such as chaos on-off keying (COOK) or differential chaos shift keying (DCSK). While COOK stands out in the area of power consumption, DCSK excels when it comes to its performance in noisy and multipath fading channels. This paper evaluates a combination of both schemes for autonomous indoor sensors. The simulation results show $50 \%$ less power consumption than DCSK and more than 3dB SNR gain in Rayleigh fading channels at BER $=10^{-3}$ as compared to COOK, making it a promising candidate for low power transmission in autonomous wireless indoor sensors. We further present an enhanced version of this scheme showing another $1 \mathrm{~dB}$ SNR improvement, but at $25 \%$ less power consumption than DCSK.
\end{abstract}

Key words: Wireless indoor sensors, low power transmission, chaotic modulation, COOK, DCSK.

\section{Introduction}

Long transmission range, low power consumption and high performance in multipath fading channels are important requirements for indoor wireless sensing [1-3]. A widely used technology for wireless sensing nowadays is zigbee [4]. Although better than other technologies like Bluetooth and Wi-Fi in terms of range and power consumption, its performance in noisy and multipath fading channels still needs optimization [1]. During the past few years, many proposals have been presented concerning chaotic carrier modulation in digital communication systems. Differing from the conventional periodical carrier modulation, chaos-based communication methods use non-periodic and broadband chaotic signals, which result in good correlation character and robust multipath performance.

Various chaos modulation schemes have been proposed, such as chaos shift keying (CSK) [5], differential chaos shift keying (DCSK) [6], chaotic on-off keying (COOK) [7] and chaotic parameter modulation (CPM) [8]. They all employ either coherent or non-coherent detection techniques. Because of the sensitivity to initial conditions, it is difficult to reliably synchronize chaotic signals at the receiver, leading to a preference for non-coherent chaos communication systems. Among several noncoherent systems proposed, one of the best biterror rate (BER) performances has been achieved by DCSK and its variation utilizing frequency modulation, FM-DCSK [9].

In DCSK, the information is transmitted in binary form. For each bit a piece of chaotic waveform is transmitted and repeated once. If the bit is a "0", the repeated waveform is changed in sign; if the bit is a "1", the waveform is simply repeated without sign change. Research on DCSK has mainly focused on increasing the transmission rate and improving the bit-error-rate in fading channels [10-13]. COOK offers the simplest solution to chaos communications. The radiation of a chaotic signal is disabled for " 0 ", and enabled for " 1 ", leading to reduced power needs compared to DCSK. As we mentioned before, low power consumption and high performance in multipath fading channels are both crucial for indoor wireless sensing. In this paper, two chaotic modulation schemes are proposed that achieve these goals by effectively combining both schemes, DCSK and COOK. The first scheme is called Differential Chaotic On-Off Keying (DCOOK), the second one is an enhanced version of it with reduced BER and called Enhanced Differential Chaotic On-Off Keying (EDCOOK). In the rest of the paper we present 
both schemes, their simulated performance and compare them to other modulation schemes.

\section{Differential Chaos On-Off Shift Keying - DCOOK}

Fig. 1 shows the transmitter and receiver structures of the proposed DCOOK system.

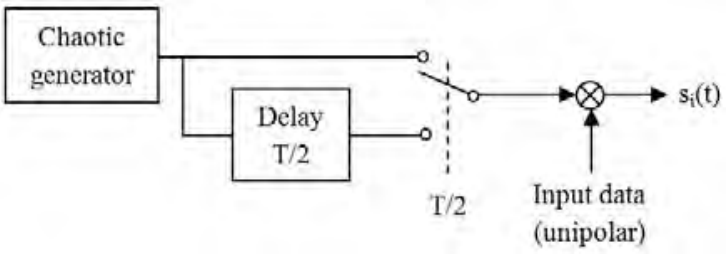

(a)

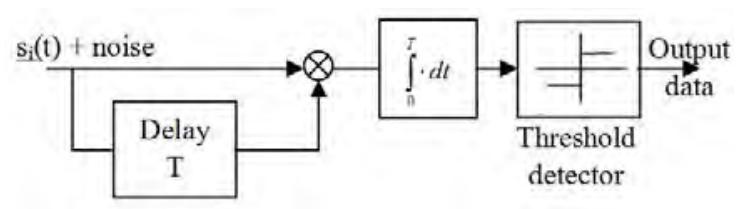

(b)

Fig. 1. Block diagram of the DCOOK system (a) transmitter (b) receiver.

At the transmitter, when the data bit is " 1 " a reference chaotic waveform $c(t)$ is transmitted during the first half of the data bit duration $T$, while a delayed version of $c(t)$ by $T / 2$ is transmitted during the second half. If the data bit is " 0 ", the transmission is disabled. We can thus write the expression for the signal transmitted by DCOOK during one symbol period $T$ as

$s_{i}(t)=\left\{\begin{array}{ll}\sqrt{E_{b}} c(t) & \text { for } d(t)=1 \text { and } 0 \leq t<\frac{T}{2} \\ \sqrt{E_{b}} c\left(t-\frac{T}{2}\right) & \text { for } d(t)=1 \text { and } \frac{T}{2} \leq t<T \\ 0 & \text { for } d(t)=0\end{array}\right.$,

with $d(t)$ being the transmitted data and $E_{b}$ the energy per bit. At the receiver, the signal is delayed by half a bit period and correlated with the undelayed signal. The result of the correlation

$$
\begin{aligned}
z_{i}= & \int_{\frac{T}{2}} r(t) r\left(t-\frac{T}{2}\right) d t \\
= & \int_{\frac{T}{2}}\left[s_{i}(t)+n_{i}(t)\right]\left[s_{i}\left(t-\frac{T}{2}\right)+n_{i}\left(t-\frac{T}{2}\right)\right] d t \\
= & \int_{\frac{T}{2}} s_{i}(t) s_{i}\left(t-\frac{T}{2}\right) d t+\int_{\frac{T}{2}} n_{i}(t) s_{i}\left(t-\frac{T}{2}\right) d t+ \\
& \int_{\frac{T}{2}} s_{i}(t) n_{i}\left(t-\frac{T}{2}\right) d t+\int_{\frac{T}{2}} n_{i}(t) n_{i}\left(t-\frac{T}{2}\right) d t
\end{aligned}
$$

is then compared with a presetted threshold to get the output decision, with $n_{i}(t)$ being the received AWGN noise. The result of the first summand in eq. 2 leads to a detected energy per bit which nearly equals $E_{b}$ in case of a transmitted ' 1 ' and zero in case of a transmitted '0':

$$
\int_{\frac{T}{2}} s_{i}(t) s_{i}\left(t-\frac{T}{2}\right) d t= \begin{cases}\int_{\frac{T}{2}} s_{i}^{2}(t) d t & \text { for } d(t)=1 \\ 0 & \text { for } d(t)=0\end{cases}
$$

The second and third summands of eq. 2 are integrals of the chaotic and noise signals, which are both statistically independent and thus delivering very low values. The last summand of eq. 2 determines the correlation of the noise at two different time epochs, also leading to very small values.

The correlator output in eq. 2 is also valid for the DCSK scheme with the difference that the data bits are bipolar, which leads to energies of $\pm E_{b}$ for ' 0 ' and ' 1 '. Therefore, we expect the BER performance of DCSK to be better than that of DCOOK, but at higher power consumption levels.

The COOK scheme uses an energy detector (square law device plus integrator) instead of differential correlation for detection, leading to a detector output:

$$
\begin{aligned}
z_{i} & =\int_{T} r^{2}(t) d t=\int_{T}\left[s_{i}(t)+n_{i}(t)\right]^{2} d t \\
& =\int_{T} s_{i}^{2} d t+\int_{T} 2 n_{i}(t) s_{i}(t) d t+\int_{T} n_{i}^{2}(t) d t .
\end{aligned}
$$

While the results of the first and second summands are similar to them of eq. 2 , the last summand would produce always positive results which are higher than those generated in eq. 2 :

$\int_{T} n_{i}^{2}(t) d t>\int_{\frac{T}{2}} n_{i}(t) n_{i}\left(t-\frac{T}{2}\right) d t$

This would result in an improvement in the BER performance of the proposed DCOOK scheme over COOK, compromising the advantages of both, DCSK with improved BER performance and $\mathrm{COOK}$ with reduced power consumption.

\section{Enhanced Differential Chaos On-Off Shift Keying - EDCOOK}

It has been shown that the BER performance of differential chaotic modulation schemes improve as the product of data bandwidth and duration $B T$ increases [8]. In mentioned DCOOK scheme we used a half bit for the reference and a half bit for the information signal and conveyed the energy over a half bit. This basically means that the performance of 
DCOOK can still be improved. One way is to combine the reference signal with the information signal, to confirm the transmitted information according to the result of a relevant operation, and to increase the integral time of the relevant testing to $1 \mathrm{bit}$, thus raising the data transmission rate under the same valuation error, as shown in Fig. 2.

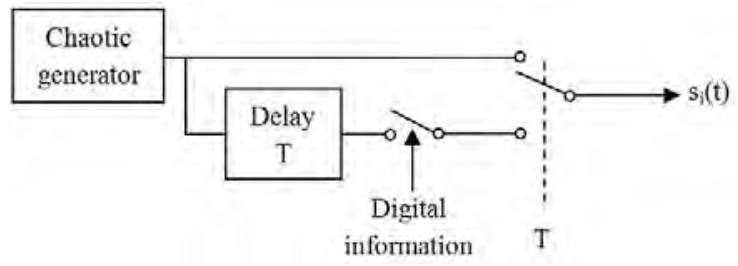

(a)

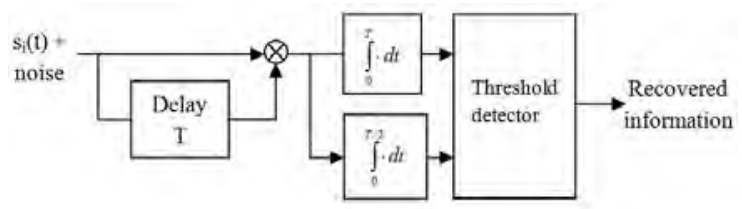

(b)

Fig. 2. Block diagram of the EDCOOK system (a) transmitter (b) receiver.

The corresponding transmitted signal

$$
s_{i}(t)= \begin{cases}\sqrt{E_{b}} c(t) & 0 \leq t<T \\ \sqrt{E_{b}} c(t-T) & \text { for } d_{1}(t)=1 \text { and } T \leq t<\frac{3 T}{2} \\ 0 & \text { for } d_{1}(t)=0 \text { and } T \leq t<\frac{3 T}{2} \\ \sqrt{E_{b}} c(t-T) & \text { for } d_{2}(t)=1 \text { and } \frac{3 T}{2} \leq t<2 \mathrm{~T} \\ 0 & \text { for } d_{2}(t)=0 \text { and } \frac{3 T}{2} \leq t<2 \mathrm{~T}\end{cases}
$$

depends now on the two adjacent data bits $d_{1}(t)$ and $d_{2}(t)$. We first send the reference signal in the first symbol and code two adjacent bits in the second symbol.

At the demodulator, the received signal is delayed by the symbol duration $T$ and correlated with the undelayed version, increasing the correlation length from $T / 2$ to $T$. In order to discern between a sent ' 01 ' and a sent ' 10 ' a $T / 2$ correlator is added in parallel. The threshold detector then decides on the values of the data pairs.

Table 1 compares the correlation length and the power saving of the proposed EDCOOK modulation scheme with other existing schemes. The advantage of a longer correlation is obtained at the expense of a reduced power saving from $50 \%$ to $25 \%$ compared to DCSK.
Tab. 1: Power saving and correlation length of EDCOOK as compared to COOK, DCSK and DCOOK.

\begin{tabular}{|c|c|c|}
\hline $\begin{array}{c}\text { Modulation } \\
\text { scheme }\end{array}$ & $\begin{array}{c}\text { Power } \\
\text { saving }\end{array}$ & $\begin{array}{c}\text { Correlation length } \\
\text { at demodulator }\end{array}$ \\
\hline DCSK & $0 \%$ & T/2 \\
\hline COOK & $50 \%$ & N/A \\
\hline DCOOK & $50 \%$ & $T / 2$ \\
\hline EDCOOK & $25 \%$ & $T$ \\
\hline
\end{tabular}

\section{Simulation Results}

We simulated a DCOOK as well as a COOK and a DCSK system for performance comparison and used Hennon mapping given by

$y_{n+1}=1-1.4 y_{n}+0.3 y_{n}^{2}$

to generate the chaotic signal. A bit rate of $T=4$ $\mu$ sec and a chip rate of $0.05 \mu \mathrm{s}$ was chosen as in [14]. Fig. 3 shows the results for an AWGN channel.

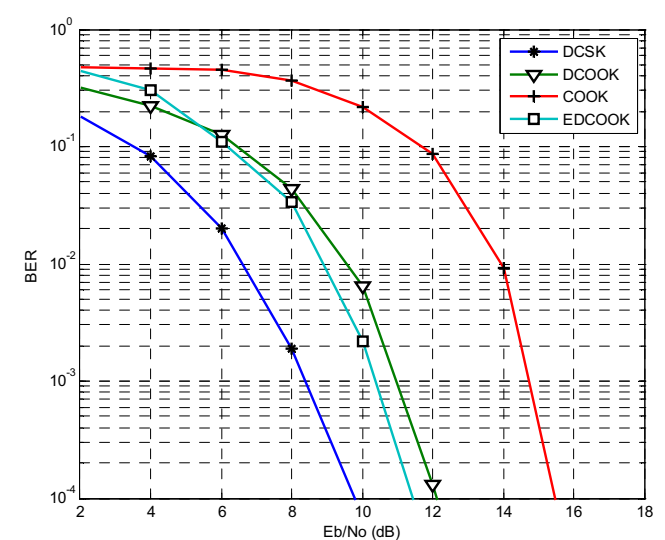

Fig. 3. BER performance of DCOOK and EDCOOK as compared with COOK and DCSK in an AWGN channel.

The noise performance of the proposed DCOOK scheme is superior to that of COOK for all signal-to-noise ratio values. The gain in signal-to-noise ratio at $P_{e}=10^{-3}$ amounts to 3.6 $\mathrm{dB}$ and lags behind the DCSK scheme by about $2 \mathrm{~dB}$. Compared to the performance of the enhanced version, the EDCOOK scheme exceeds the original DCOOK scheme for signal-to-noise ratio values greater than $5 \mathrm{~dB}$ by less than $1 \mathrm{~dB}$ at $P_{e}=10^{-3}$.

Fig. 4 finally shows the performance in Rayleigh fading channels with two paths, where the second path was delayed by 75 ns and attenuated by $-3 \mathrm{~dB}$ in order to approximately simulate the multipath environment inside an office building. The performance of DCOOK is once again better than COOK at all signal-tonoise ratio values. The gain in signal-to-noise 
ratio at $P_{e}=10^{-3}$ amounts to $3 \mathrm{~dB}$ and lags behind the DCSK scheme by about $1.5 \mathrm{~dB}$.

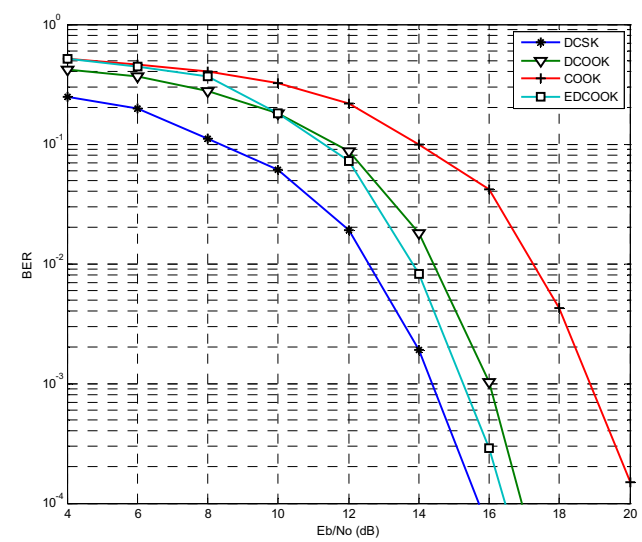

Fig. 4. BER performance of DCOOK and EDCOOK as compared with COOK and DCSK in Rayleigh fading channels.

The simulation also shows that the EDCOOK scheme has a relatively better BER performance than DCOOK for signal-to-noise ratio values higher than $10 \mathrm{~dB}$. The existence of this threshold is due to the greater space of the decision variable in the case of DCOOK as compared to EDCOOK showing a larger effect on the BER performance than the length of correlation at lower signal-to-noise ratio values.

\section{Conclusions}

Two chaotic communication schemes have been presented: differential chaos on-off keying DCOOK and its enhanced version EDCOOK. They were designed to combine the advantages of DCSK and COOK for multipath fading channels and low power consumption. The DCOOK scheme increases the signal space of the decision variable by using the concept of differential correlation in order to increase the BER performance and reduces the power consumption by $50 \%$. The enhanced version EDCOOK improves the performance of DCOOK further but reduces the saving of power consumption to $25 \%$. This is obtained by increasing the correlation length through sending a pair of bits with one being the reference chaotic signal. The selection of the DCOOK scheme or its enhanced version depends on the demands of the wireless sensing communication system designer.

\section{References}

[1] M. Brambley, M. Kintner-Meyer, S. Katipamula, , P. O'Neill, Wireless Sensor Applications for Building Operation and Management, Web Based Energy Information and Control Systems: Case Studies and Applications, chap. 27, eds. B.L. Capehart, L.C. Capehart, Fairmont Press/CRC Press, Lilburn GA, 341-367 (2005)
[2] S. K. Palit, Design of Wireless Communication Sensing Networks for Tunnels, Trains and Buildings, International Journal on Smart Sensing and Intelligent Systems, vol.2, no.1, 118-134 (2009)

[3] W. Su, O. Akun, E. Conant, Communication Protocols for Sensors and Networks, Wireless Sensor Networks, eds. C. S. Raghavendra, K. M. Sivalingam , T. Znati, Kulwer Academic Publishers, Boston, 21-50 (2004)

[4] A. Elahi, A. Gschwender, ZigBee Wireless Sensor and Control Networks, Prentice Hall Inc., USA, 2010.

[5] H. Dedieu, M. P. Kennedy, M. Hasler, Chaos shift keying: modulation and demodulation of a chaotic carrier using self-synchronizing Chua's circuits, IEEE Transactions on circuits and systems II, vol. 40, 10, 634-643 (1993)

[6] G. Kolumbán, M. P. Kennedy, G. Kis, Performance improvement of chaotic communications systems, Proceedings of European Conference on Circuit Theory and Design, 84-89 (1997)

[7] G. Kolumbán, G. K. Vizvari, W. Schwarz, Differential chaos shift keying: a robust coding for chaos communication, Proceedings of International Workshop on Nonlinear Dynamics of Electronic Systems, 92-97 (1996)

[8] G. Kolumbán, M. P. Kennedy, , L. O. Chua, "The role of synchronization in digital communications using chaos-part II: chaotic modulation and chaotic synchronization," IEEE Trans. Circuits Syst.-I: Fundamental Theory Appl., vol. 45, no. 11, Nov. 1998.

[9] G. Kolumbán, G. Kis, Z. Jákó, M. P. Kennedy, FMDCSK: A Robust Modulation Scheme for Chaotic Communications, IEICE Transactions on the Fundamentals, vol. E81-A, 1798-1802 (1998)

[10] J. Pan, H. Zhang, Design of FM-QACSK Chaotic Communication System, International Conference on Wireless Communications \& Signal Processing, Nanjing, 1184-1187 (2009)

[11] S. Zhu, Y. Xu, K. Yin, Design of a Quadrature Differential Chaotic Phase Shift Keying Communication System, Proceedings of the International Conference on Networks security, Wireless Communications and Trusted Computing, 518-521 (2009)

[12] H. Abdullah, Design of High data Rate FM-QCSK Chaotic Communication System, Journal of Wireless Networking and Communications, vol.2, 4, 49-54 (2012)

[13] H. Jian-Qun, Z. Yi-Sheng, A Method of Raising the Transmission Effieciency in DCSK, Proceedings of 2005 IEEE International Symposium on Microwave, Antenna, Propagation and EMC Technologies for Wireless Communications, 1307-1310 (2005)

[14] M. A. B. Faran, A. Kachouri, M. Samet, Design of Secure Digital Communication Systems using DCSK Chaotic Modulation, DTIS 2006 International Conference on Design and Test of Integrated Systems in Nanoscale Technology, Tunis, 200-204 (2006) 